\title{
Inferior part of rectus abdominis muscle flap: A case report
}

\author{
Mahdi Alemrajabi*1, Saeed Moradi², Sepideh Jahanian², Behrouz Banivaheb², Nima Hemmati², \\ Saeed Safari²
}

\section{Abstract}

Background: Abdominoperineal resection (APR) is the standard surgical treatment for low-lying anorectal malignancies. It seems that immediate flap reconstruction has fewer complications compared to primary closure. There are several options for local flap reconstruction of perineal wound closure, and each specific flap method has its own advantages and disadvantages.

Case presentation: In this case report, a new method of reconstruction is presented which contains only the inferior part of the rectus abdominis muscle in 2 patients, one with unilateral and the other with bilateral involvement and they both underwent APR. Both patients were referred to the colorectal surgery clinic for APR by an oncologist. Both patients had severe constipation and both reported pain on defecation and rectorrhagia. Patient 1 received a unilateral inferior part of rectus abdominis muscle flap and patient 2 received a bilateral flap.

Conclusion: Immediate flap reconstruction after APR has fewer complications than primary closure and the inferior part of rectus abdominis muscle flap seems to be a possible means of reconstruction after APR.

Keywords: Muscle flaps, Surgical flaps, Abdominoperineal resection, Rectal malignancy, Case-report, Reconstructive surgical procedures

Conflicts of Interest: None declared

Funding: None

*This work has been published under CC BY-NC-SA 1.0 license

Copyright $\odot$ Iran University of Medical Sciences

Cite this article as: Alemrajabi M, Moradi S, Jahanian S, Banivaheb B, HemmatiN, Safari S. Inferior part of rectus abdominis muscle flap: A case report. Med J Islam Repub Iran. 2019 (16 Oct);33:111. https://doi.org/10.47176/mjiri.33.111

\section{Introduction}

Abdominoperineal resection (APR) is the standard surgical treatment for low-lying anorectal malignancies. Large resections usually result in an extensive pelvic defect associated with complications such as wound closure challenges and infections. It seems that immediate flap reconstruction has fewer complications compared to primary closure (1-5). There are several options for local flap reconstruction of perineal wound closure, which include pedicled vertical rectus abdominis myocutaneous (VRAM) flaps, local V to Y advancement flaps, and pedicled gracilis muscle flaps. However, as each specific flap method has its own advantages and disadvantages, choosing the best flap method is challenging (6-9). Maintaining

Corresponding author: Dr Mahdi Alemrajabi, alemrajabi.m@iums.ac.ir

1. Department of Surgery, Firoozgar Clinical Research Development Center (FCRDC), Iran University of Medical Sciences, Tehran, Iran

2. Firoozgar Clinical Research Development Center (FCRDC), Iran University of Medical Sciences, Tehran, Iran the advantages of previous methods while eliminating disadvantages can be a significant step forward in providing better care for patients.

In this case report, a new method of reconstruction was presented which involved only the inferior part of the rectus abdominis muscle in 2 patients, one unilateral and the other bilateral; both patients underwent APR. Flaps are usually employed in perineal wound reconstruction, but they are also applicable in pelvic inlet closure to protect the bowels from herniation and entrapment.

\section{Case report}

Patient 1 was a white 64-year-old male elementary $\uparrow$ What is "already known" in this topic:

Immediate flap reconstruction has fewer complications compared to primary closure after abdominopelvic resection. Flap reconstruction is associated with its own unique complication and advantages.

\section{$\rightarrow$ What this article adds:}

The inferior part of rectus abdominis muscle flap seems to be a possible reconstruction method after abdominopelvic resection with possible fewer complications than similar reconstructive methods. 
school teacher, and patient 2 was a white 58-year-old male farmer. Both patients were referred to the colorectal surgery clinic for APR surgery by an oncologist following the diagnosis of rectal cancer. The initial presentation was constipation for patient 1 and severe weight loss for patient 2. Both patients had severe constipation and both reported pain on defecation and rectorrhagia. There was no history of prior surgeries, allergic reaction to drugs, psychological diseases, and smoking. A mass was found in the digital rectal examination in both patients, and they both underwent neoadjuvant chemoradiotherapy to shrink the tumor size.

Pathologic studies revealed rectal adenocarcinoma. Endoanal sonographic study showed distal rectal mass with involvement of external anal sphincter in both patients. There was no metastasis in patients. All options alongside the new method were described to the patients and informed written consent was obtained.

The patients were positioned in a semi-lithotomy position and received general anesthesia. Ceftriaxone and metronidazole were administered intravenously at the induction of anesthesia. The patients also received neomycin and metronidazole orally a day before surgery and underwent APR surgery. Inferior part of rectus abdominis muscle flap was used for reconstruction with the following technique (Alem Method).

For patient 1, the flap was taken from the inferior part of

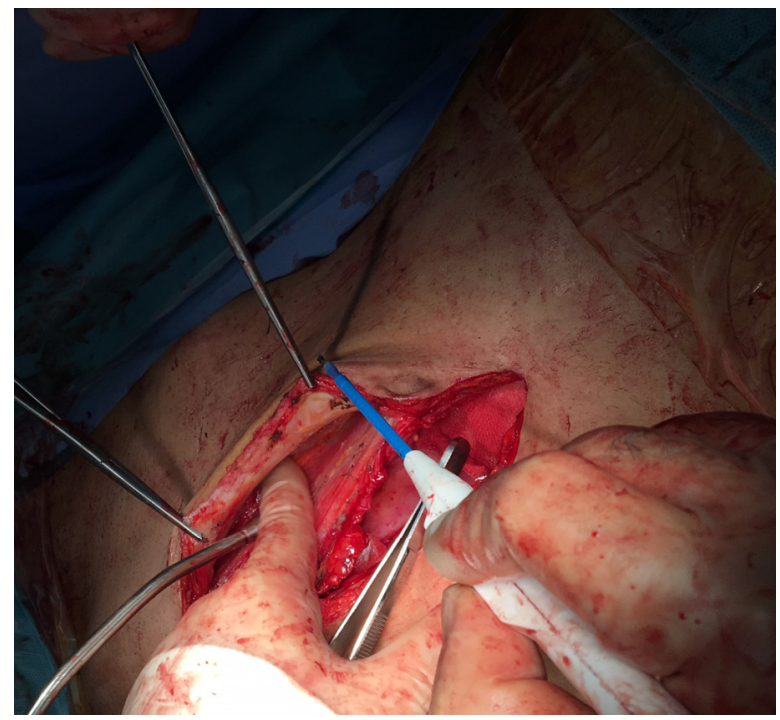

Fig. 1. The inferior part of the rectus abdominis muscle is dissected from the anterior fascia and skin

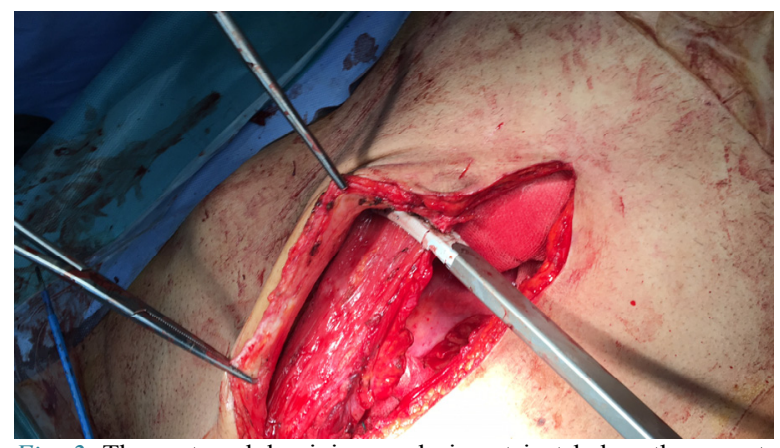

Fig. 2. The rectus abdominis muscle is cut just below the arcuate line the rectus abdominis muscle and mobilized by lifting up the skin paddle, its underlying fat, and rectus muscle. The skin and anterior fascia were dissected from the bulk of the muscle (Fig. 1). The right rectus abdominis muscle was preferably selected to allow colostomy through the left rectus muscle. The inferior part of rectus abdominis muscle was cut just below the arcuate line and medial to the semilunar line (Fig. 2). The inferior epigastric artery was carefully dissected. Perforating vessels in anterior rectus sheath were also dissected to achieve minimal fascial resection to prevent abdominal hernias and allowing primary closure of the defect. The flap dissection was continued to the origin which is on the pubis (Fig. 3). The flap was then fashioned into the perineum (Fig. 4). The skin and external rectus fascia was used to repair the abdominal incision, so the risk of an incisional hernia was minimized and the muscle itself was used to fill the defect in the pelvic floor. Multiplelayer sutures with Vicryl 2-0 were used to place the flap without any tensions (Fig. 5). The defect created by the rectus muscle excision on the abdominal wall was closed and repaired primarily with minimal tension using nylon. To reduce the risk of infection, no mesh was used.

The same procedure was done for patient 2, but in both

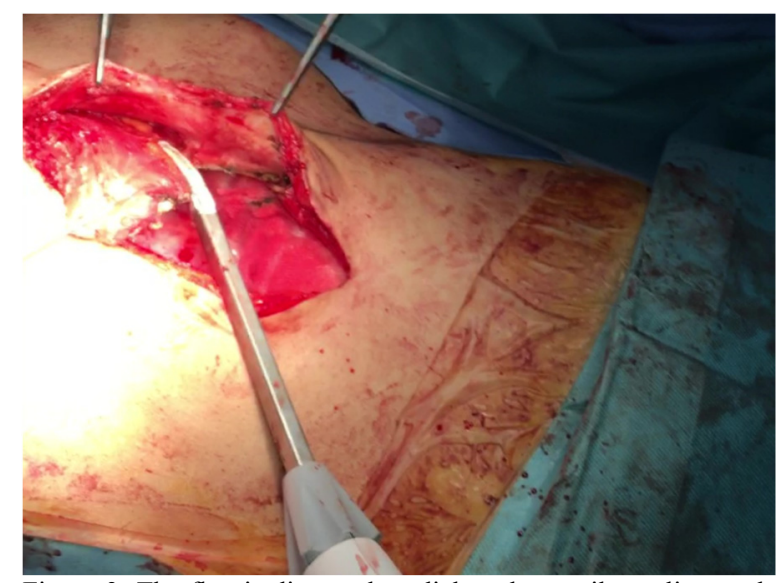

Figure 3. The flap is dissected medial to the semilunar line to the pubic insertion of the muscle

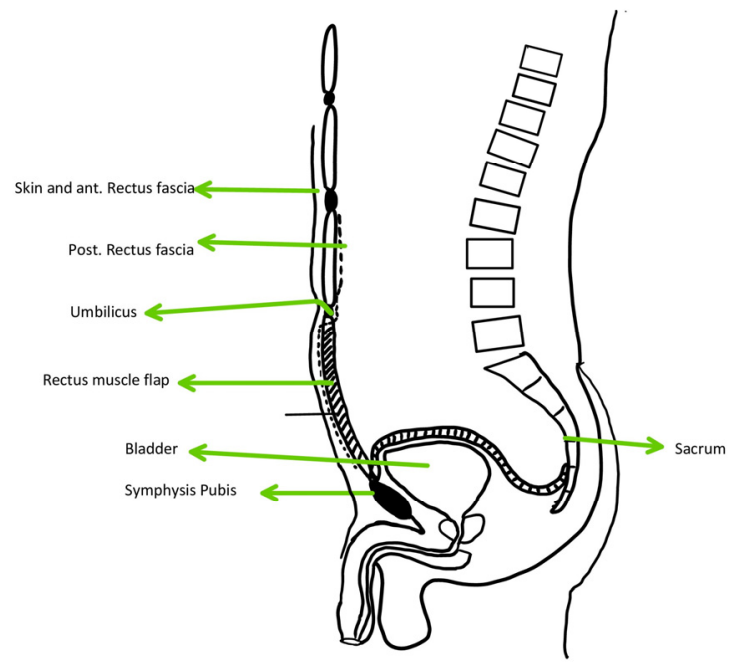

Fig. 4. The flap rotation and relocation to fill the defects of the pelvis 


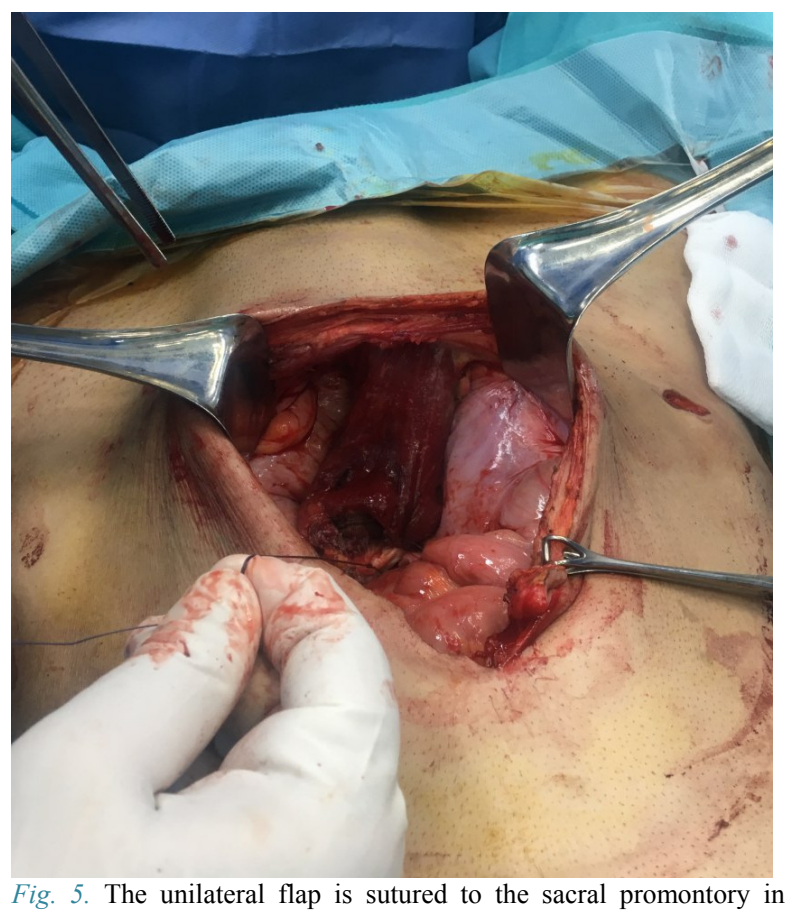
multiple layers to minimize the tension

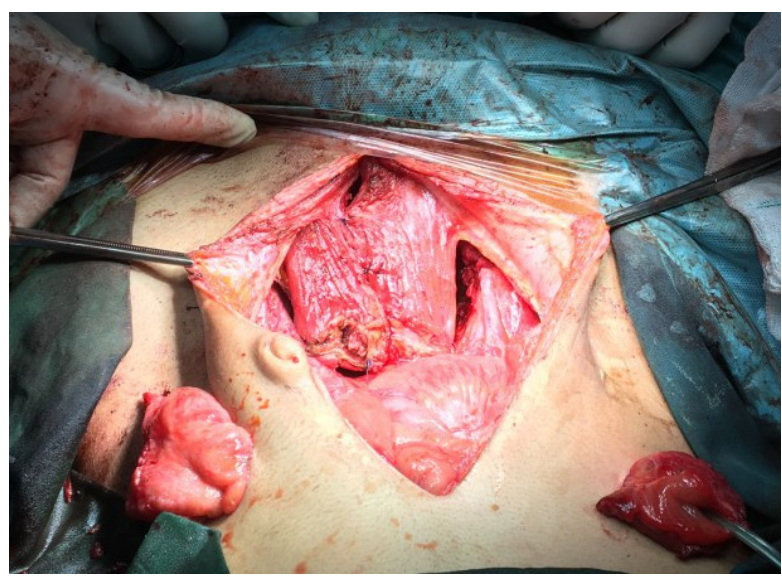

Fig. 6. The bilateral flap is sutured to the sacral promontory in multiple layers to minimize the tension

sides, which resulted in a bilateral inferior rectus abdominis muscle flap reconstruction, since the pelvic cavity of patient 2 could not be covered by a unilateral flap. The colostomy in this patient was placed on the left side of the abdomen (Fig. 6).

After the surgery, 5000 units of heparin was administered twice a day subcutaneously.

This procedure was done on a human in this study for the first time, and an attending colorectal surgeon with more than 6 years of experience performed the procedure.

The follow-up visits were scheduled for 1 week, 1 month, 3 months, 6 months, and 12 months after the surgery in the colorectal surgery clinic.

The duration of surgery was 124 minutes for patient 1 and 142 minutes for patient 2. After the surgery, colostomy functioned properly in both patients. The length of hospital stay for patient 1 was 5 days and for patient 2 was 4 days. Both patients started walking and taking liquid food 1 day and 2 days after the surgery, respectively.
The patients were instructed about the ostomy care and other postabdominal surgery cares (heavy lifting and abdominal binder).

No significant blood loss was observed during the surgery. Patient 1 had an abdominal wound infection, with mucopurulent discharge, which was treated conservatively with orally administered antibiotics, wound washing, and changing clothes twice a day (Clavian-Dindo grade I). There was no specific complication in patient 2 after the surgery and before discharge. In the follow-up period, there were no reported or observed complications in the patients. No fatal outcomes were reported in the 12-month follow-up period. Obstruction, wound infection, abdominal hernia, abscess formation, flap loss, cellulitis, prolonged healing, pulmonary thromboembolism, deep vein thrombosis, parastomal hernias, and urinary tract infection were not observed.

\section{Discussion}

There were no flap-related complications in this study. One of the advantages of using the inferior part of the rectus abdominis as a flap is that it provides enough muscle bulk to fill the vacant space after APR. Since only the inferior part is used as a flap, there may be less abdominal wall morbidity compared to other techniques.

This was a case report and had many limitations. None of the 2 cases were female; in female patients the pelvic inlet can be closed by the uterus. Both patients received chemoradiotherapy prior to the surgery. The follow-up period for this study was 12 months. Flap loss and mortality are 2 potential complications associated with this method if it is applied to a larger population.

In different surgeries, using a myocutaneous flap is better than primary closure considering complications and outcomes $(4,10)$. Primary closure is associated with different complications such as wound dehiscence $(5,11)$. There are other alternatives to this method, the best of which seems to be VRAM flaps. Sheckter and et al compared 3 different reconstruction methods and found that the V-to-Y advancement reconstruction had higher wound-healing complications, with a rate of $73.03 \%$. Also, in their study, the complication rate in the gracilis flap group was $75 \%$. The best reconstruction method in the study was VRAM flaps, with the complication rate of $22.2 \%$; however, only 9 patients were observed in the VRAM flap group (12). The VRAM flap is considered a reliable and safe method for immediate reconstruction in patients undergoing APR. It is claimed that the overall complication rate in the VRAM flap reconstruction is $11 \%$; however, the complication rate can vary based on the stage of malignancy. For example, in the Horch R.E. et al study, a higher complication rate of $22.8 \%$ was observed in the patients with higher stages of malignancy (stages III and IV), but the complication rate in stage I and II patients was $12.96 \%$ (13). Since both patients in the present study were at stage II of the disease, a low complication rate was expected. No mortality was recorded in the hospital after the surgery and before discharge. These results are also comparable with those of other studies. In a study by Hinijosa and $M$. et al on patients with rectal 
tumors undergoing APR with VRAM flaps, no mortality was recorded in the 30-day hospital stay (14). In another study with the aim of examining the survival and complications of anal cancer using VRAM flaps, the overall long-term survival was calculated to be $66 \%$. Since the inferior part of the rectus abdominis muscle flap is smaller than that of VRAM flaps, the abdominal wall morbidity seems to be much less frequent in this technique. Also, because in the one-sided flap, the right rectus abdominis muscle was selected as the flap, there were no limitations on providing ostomies for the patients, a well-known problem in VRAM flaps. Patient 2 who received 2-sided inferior rectus abdominis muscle flap developed ostomy complication after 6 months, which could be due to less support for ostomy placement. Although the muscle flap was less bulky than that of VRAM, it provided enough bulk to fill the dead space (Figs. 1 and 6 . The arc of the motion of this flap seems to be comparable with that of gracilis flaps and this kind of flap can provide enough space to cover for the pelvic defects (15).

In conclusion, immediate flap reconstruction after APR has fewer complications than primary closure and the inferior part of rectus abdominis muscle flap seems to be a possible reconstruction method after APR.

\section{Conflict of Interests}

The authors declare that they have no competing interests.
Closure After Abdominal Fascial Dehiscence Using Nasogastric Tube and Hemovac Perforator: A Case-Series Study. World J Surg. 2018;42(10):3106-11.

12. Sheckter CC, Shakir A, Vo H, Tsai J, Nazerali R, Lee GK. Reconstruction following abdominoperineal resection (APR): Indications and complications from a single institution experience. J Plast Reconstr Aesthet Surg. 2016;69(11):1506-12.

13. Horch RE, Hohenberger W, Eweida A, Kneser U, Weber K, Arkudas A, et al. A hundred patients with vertical rectus abdominis myocutaneous (VRAM) flap for pelvic reconstruction after total pelvic exenteration. Int J Colorectal Dis. 2014;29(7):813-23.

14. Hinojosa MW, Parikh DA, Menon R, Wirth GA, Stamos MJ, Mills $\mathrm{S}$. Recent experience with abdominal perineal resection with vertical rectus abdominis myocutaneous flap reconstruction after preoperative pelvic radiation. Am Surg. 2009;75(10):995-9.

15. Ducic I, Dayan JH, Attinger CE, Curry P. Complex perineal and groin wound reconstruction using the extended dissection technique of the gracilis flap. Plast Reconstr Surg. 2008;122(2):472-8.

\section{References}

1. Chan S, Miller M, Ng R, Ross D, Roblin P, Carapeti E, et al. Use of myocutaneous flaps for perineal closure following abdominoperineal excision of the rectum for adenocarcinoma. Colorectal Dis. 2010;12(6):555-60.

2. Wang ED, Conkling N, Xu X, Chern H, Finlayson E, Varma MG, et al. Perineal flap reconstruction following oncologic anorectal extirpation: an outcomes assessment. Plast. Reconstr. Surg. 2015;135(1):176e-84e.

3. Howell AM, Jarral OA, Faiz O, Ziprin P, Darzi A, Zacharakis E. How should perineal wounds be closed following abdominoperineal resection in patients post radiotherapy--primary closure or flap repair? Best evidence topic (BET). Int J Surg. 2013;11(7):514-7.

4. Johnstone MS. Vertical Rectus Abdominis Myocutaneous Versus Alternative Flaps for Perineal Repair After Abdominoperineal Excision of the Rectum in the Era of Laparoscopic Surgery. Ann Plast Surg. 2017;79(1):101-6.

5. Devulapalli C, Jia Wei AT, DiBiagio JR, Baez ML, Baltodano PA, Seal SM, et al. Primary versus Flap Closure of Perineal Defects following Oncologic Resection: A Systematic Review and MetaAnalysis. Plast Reconstr Surg. 2016;137(5):1602-13.

6. Kolehmainen M, Suominen S, Tukiainen E. Pelvic, perineal and genital reconstructions. Scand J Surg. 2013;102(1):25-31.

7. Persichetti P, Cogliandro A, Marangi GF, Simone P, Ripetti V, Vitelli $\mathrm{CE}$, et al. Pelvic and perineal reconstruction following abdominoperineal resection: the role of gracilis flap. Ann Plas Surg. 2007;59(2):168-72

8. Carey JN, Sheckter CC, Watt AJ, Lee GK. Intra-abdominal pedicled rectus abdominis muscle flap for treatment of high-output enterocutaneous fistulae: case reports and review of literature. J Plast Reconstr Aesthet Surg. 2013;66(8):1145-8.

9. Nelson RA, Butler CE. Surgical outcomes of VRAM versus thigh flaps for immediate reconstruction of pelvic and perineal cancer resection defects. Plast Recontr Surg. 2009;123(1):175-83.

10. Azizi R, Alemrajabi M. Trends in Surgical Treatment of Pilonidal Sinus Diseases: Primary Closure or Flap? World J Surg. 2012;36(7):1713-4

11. Vahedian J, Jahanian S, Banivaheb B, Hemmati N, Ghavamipour M, Chegini M, et al. A New Method for Surgical Abdominal Mass 